Article

\title{
Demonstration of Bomb Detection Using the IBM Quantum Computer
}

\author{
Ankit Raj ${ }^{1, \ddagger}$, Barnali Das ${ }^{1, \ddagger}$, Bikash K. Behera ${ }^{1}$ and Prasanta K. Panigrahi ${ }^{1, *}$ \\ 1 Department of Physical Sciences, \\ Indian Institute of Science Education and Research Kolkata, Mohanpur 741246, West Bengal, India; \\ ar18ms210@iiserkol.ac.in; bd18ms201@iiserkol.ac.in; bkb18rs025@iiserkol.ac.in; pprasanta@iiserkol.ac.in \\ * Correspondence: pprasanta@iiserkol.ac.in; Tel.: +91-974-891-8201 \\ $\ddagger$ These authors contributed equally to this work.
}

\begin{abstract}
According to Copenhagen interpretation, a quantum particle can exist in a superposition of all possible states, out of which only one state is observed when it is measured. Interestingly, it has been observed that interaction with the quantum particle during measurement can also affect the outcome of the state. A scheme for interaction free measurement was proposed by Elitzur and Vaidman [Found. Phys. 23, 987 (1993)], where they used Mach Zehnder interferometer to detect whether a bomb is alive or dead. In $25 \%$ of the cases they were able to detect that the bomb is alive without exploding it. Here, we demonstrate the above experiment using quantum computing, which can be realized in a quantum computer designing quantum circuits on it. We explicate all the cases, including whether the bomb is alive or dead by proposing new quantum circuits and executing those in QISKit as provided by IBM Quantum Experience platform and verify the obtained results.
\end{abstract}

Keywords: Bomb Detection; Interaction Free Measurement; Mach Zhender Interferometer; IBM Quantum Expeience

\section{Introduction}

The meaning of the word "interaction-free" is that there is no interaction with an object. In case of a bomb, it is quite obvious that explosion means interaction and no interaction means no explosion. Classical physics unequivocally supports this argument but quantum mechanics has its own beautiful way of disproving this. This paves the way to the Interaction Free Measurement (IFM) which has several experimental realizations [1-10] like in novel quantum non-demolition techniques $[4,11]$ for improving cryptographic schemes [12,13] and even for "interaction-free" computation [14]. After Elitzur and Vaidman (EV) IFM proposal [15] further progress in this field leads to higher efficiency [16] Interaction free measurement. In Kwait et al. experiment [1], they used quantum Zeno effect to achieve almost $100 \%$ efficient scheme. Another modification of EV IFM which leads to the efficiency of almost $100 \%$ that has been proposed by Paul and Pavicic [17] and implemented in a laboratory by Tsegaye et al. [4]. The basic concept in their experiment involves optical resonance cavity which is almost transparent when empty and is almost perfect mirror when there is an object inside. Other modifications of IFM are related to interaction free imaging [7] and interaction free measurement of semi-transparent objects. All these experiments achieve an important practical goal of interaction free measurement.

The IBM Quantum Experience (IBM QE) [18] is an online platform that gives users free access to 5-qubit and 14-qubit quantum computers. It allows us to use its python-based software developer kit to write and run quantum algorithms. It has been used in numerous quantum problems for fast calculations and the ability to handle huge data. Unlike the conventional memory units, where each qubit (the smallest unit of information) is represented by a microscopic dot on a microchip in a 
quantum computer, that can be both 0 and 1 at the same time. This superposition allows quantum computers to perform operations on many values in one fell swoop instead of executing sequentially. We use QISKit (Quantum Information Software Kit) [19] to experimentally demonstrate the detection of bomb using the 5-qubit quantum chip, 'ibmqx4'. The Jupyter Notebook App [20] is used here to write the programs which contains both computer code (e.g. python) and rich text elements such as paragraph, equations, figures, links etc to make user friendly. Several quantum computational tasks have been performed using IBM quantum experience platform such as quantum machine learning [21,22], quantum simulation [23-28], quantum error correction [29-32], quantum cryptography [33,34], quantum information theory [35-37], quantum algorithms [38,39], quantum optimization problems [40], quantum games [41-43], designing quantum communication devices [44,45].

Here, we experimentally realize the above experiment using the IBM QE platform. We propose new quantum circuits and design those using QISKit to demonstrate the scheme of Elitzur and Vaidman Interaction Free Measurement. We exploit the use and application of a quantum computer which can be utilized to illustrate experiments that have been performed using other architectures such as Michelson Interferometer or other optical setups. Similar works have already been done $[34,44]$ where a quantum computer has been used to show its future application even if no experimental architectures are easily available to a researcher.

The rest of the paper is organized as follows. Section 2 briefly describes the scheme of Elitzur and Vaidman Interaction Free Measurement (EV IFM). Section 3 discusses about the operations used in the quantum circuit for the proposed experiment. In Section 4, we elaborate the quantum circuits for Elitzur and viadman model for IFM. Sections 5 and 6 introduce the cases when the bomb is live and dead respectively. Finally, we conclude in Section 7 and discuss about the future direction of the present work.

\section{Scheme of Elitzur and Vaidman Interaction Free Measurement (EV IFM)}

The Elitzur and Vaidman Interaction Free Measurement (EV IFM) is based on the historical single-photon interference experiments using Mach Zehnder interferometer. As per this setup in Fig. 3 , a quantum test particle (here it is photon that can take either horizontal or vertical path) reaches the beam splitter BS1 which has transmission coefficient $1 / 2$ and reflection coefficient $1 / 2$. The transmitted and reflected parts of the particle wave are then reflected by the mirrors (M1 and M2) and finally recombine at another beam splitter BS2 with transmission and reflection coefficient $1 / 2$. Two detectors are positioned to detect the particle after it passes through BS2. The positions of the beam splitters and the mirrors are arranged in such a way that (because of destructive interference) the particle is never detected by any one of the detectors (say D2), in case the particle chooses the vertical path. To detect a bomb this interferometer is designed in such a way that one of the two routes of the particle contains the bomb (the bomb here is an object which is quantum particle sensitive object i.e., it explodes when the particle interacts with it). There are three possible outcomes of this measurement: (i) explosion; (ii) detector D1 clicks; (iii) detector D2 clicks. The probabilities of the three outcomes are 1/2,1/4 and 1/4 respectively. In case the particle chooses the vertical path then clicking of detector D1 does not tell anything about the presence or absence of bomb since it clicks when the bomb is not there or in case of dead bomb and also has the clicking probability of $25 \%$ when the bomb is present. Explosion is an obvious method to know the presence of bomb but since it involves interaction of particle and bomb it comes out to be unnecessary to consider. The remaining $25 \%$ is the most useful of all. It is the case when D2 clicks, the goal is achieved: we know that the object is inside the interferometer and it did not explode. The EV method allows finding sensitivity of bomb without exploding it with certainty. The bomb might explode in the process, but there is at least a probability of $25 \%$ to detect the bomb without the explosion. Here "Certainty" makes sure the existence of the bomb inside the interferometer when the detector D2 clicks, i.e., the process is successful. The above process is explained considering the input of photon in the vertical direction. If the input path is taken horizontal then clicking of D1 will announce the presence of bomb. 


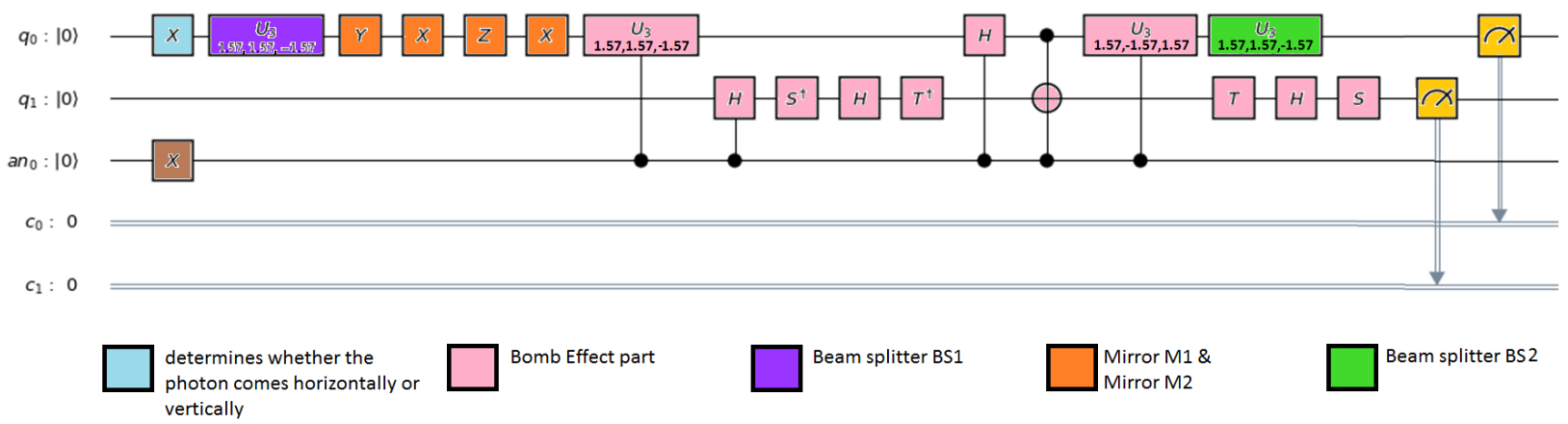

Figure 1. Quantum circuit illustrating the scheme for the bomb detection in the quantum computer. The different coloured parts represent different components of the experimental setup in terms of various gates. The absence of sky blue coloured box implies that the photon is directed from vertical direction while its presence implies that it is directed from horizontal direction. Pink coloured part represents bomb effect part, Violet coloured box represents beam splitter 1 (BS1), green box represents beam splitter 2 (BS2) and Yellow boxes are measurement boxes. The presence of brown box tells that the bomb is present.

\section{Quantum Gates used in the experiment}

The beam splitter BS1 and beam splitter BS2 in the Figs. 3, 6, 9, and 12 are defined by U3 operation. The mirrors M1 and M2 in the Figs. 3, 6, 9, and 12 are composed of sequential operation of $X, Y, Z$ and $X$ gates. The operations for the effect of bomb part are $\mathrm{U} 3, \mathrm{H}, \mathrm{S}^{\dagger}, \mathrm{T}^{\dagger}, \mathrm{U}^{\dagger}, \mathrm{S}$ and $\mathrm{T}$, whose matrix forms are explicitly given below.

$$
\begin{gathered}
X=\left[\begin{array}{ll}
0 & 1 \\
1 & 0
\end{array}\right], Y=\left[\begin{array}{cc}
0 & -i \\
i & 0
\end{array}\right], Z=\left[\begin{array}{cc}
1 & 0 \\
0 & -1
\end{array}\right], U 3=\frac{1}{\sqrt{2}}\left[\begin{array}{cc}
1 & i \\
i & 1
\end{array}\right], H=\frac{1}{\sqrt{2}}\left[\begin{array}{cc}
1 & 1 \\
1 & -1
\end{array}\right], \\
S^{+}=\left[\begin{array}{cc}
1 & 0 \\
0 & -i
\end{array}\right], T^{+}=\left[\begin{array}{cc}
1 & 0 \\
0 & \frac{1-i}{\sqrt{2}}
\end{array}\right], U 3^{+}=\frac{1}{\sqrt{2}}\left[\begin{array}{cc}
1 & -i \\
-i & 1
\end{array}\right], S=\left[\begin{array}{cc}
1 & 0 \\
0 & i
\end{array}\right], T=\left[\begin{array}{cc}
1 & 0 \\
0 & \frac{1+i}{\sqrt{2}}
\end{array}\right] .
\end{gathered}
$$

\section{Quantum circuit for interaction free measurement}

The scheme in Fig. 1 shows the setup for the quantum bomb detection with various colours representing various parts of the interferometer. We take three qubits, $q_{0}, q_{1}$ and $a n_{0}$, where the first two qubits $\left(q_{0}\right.$ and $\left.q_{1}\right)$ represent the state of the photon and the third qubit, $a n_{0}$ signifies whether the bomb is there or not. If there is $X$ gate on the qubit $q_{0}$ shown by light blue colour decides which direction the photon chooses whether horizontal or vertical, i.e., if the state of the first two qubits is $|10\rangle$, it denotes horizontal direction of the photon, if the state is $|00\rangle$, it denotes the vertical direction of the photon. In a similar way, the $\mathrm{X}$ gate on the qubit $a n_{0}$ shown by brown colour decides whether the bomb is alive or dead, i.e., the presence or absence of bomb. Here the absence of an active bomb is shown by $|0\rangle$ state and the presence of an active bomb by $|1\rangle$ state. The violet colour represents the beam splitter BS1 and the green colour represents the beam splitter BS2 which are the U3 gate with the parameters $\theta, \phi$ and $\lambda$ as $\pi / 2, \pi / 2$ and $-\pi / 2$ respectively. The mirrors M1 and M2 shown by orange colour combinedly consist of the $\mathrm{Y}, \mathrm{X}, \mathrm{Z}, \mathrm{X}$ gates and the pink coloured part consists of controlled-U3 on the qubit $q_{0}$, controlled- $\mathrm{H}, S^{\dagger}, \mathrm{H}, T^{\dagger}$ on the qubit $q_{1}$, controlled-H on the qubit $q_{0}$ then a controlled-not-controlled on the qubit $q_{1}$, controlled- $U 3^{\dagger}$ on the qubit $q_{0}$ and T, H, S on the qubit $q_{1}$ which acts as the effect of bomb. The yellow boxes are there for measurement and calculation of result in the form of probabilities as shown in Figs. 4, 7,10, and 13. It is to be carefully noted that the operations of the bomb effect part act only when live bomb is there. 


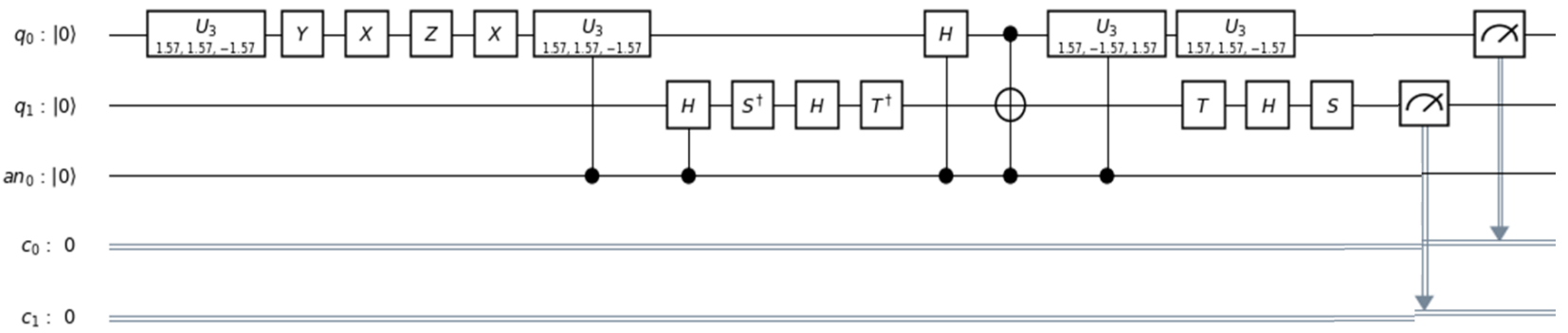

Figure 3. Quantum circuit for the case when the bomb is not alive and the input is from vertical direction. As it can be seen, there is no X gate on the qubit $q_{0}$, hence the state of the qubits $q_{0}$ and $q_{1}$ is in $|00\rangle$ state, that implies the vertical direction of the photon. The qubit $a n_{0}$ is in $|0\rangle$ state, which signifies there is no bomb in the path or the bomb is dead. Hence the part of bomb effect, i.e., the controlled operations from the qubit $a n_{0}$ to the first two qubits $q_{0}$ and $q_{1}$ would have no effect.

\section{CASE-1: No bomb or dead bomb}

\section{When the input of photon is from vertical direction}

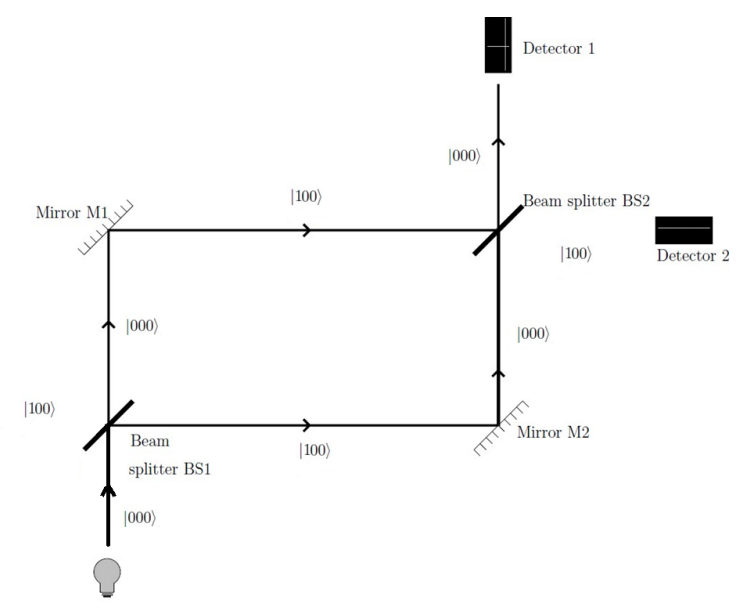

Figure 2. Setup when the bomb is not alive and the photon input is from vertical direction. In this case D1 will click and D2 will not. Constructive interference will occur at D1 while destructive interference at D2.

Here if no bomb is there then the states of the photon superpose with each other constructively and destructively at respective detectors (depending on the direction of input of the photon). And if the bomb is dead then also superposition occurs because it (the photon-sensitive bomb) allows the photon to pass through it without any disturbance. The input photon is from vertical direction (by convention discussed in scheme in the Section 4), which is taken to be $|00\rangle$ state as shown in Fig. 2. Initially, as there is no bomb, the state of the whole system is given as $|000\rangle$. On passing the photon through BS1 i.e. applying the U3 gate on the first qubit $q_{0}$ we get $\frac{(|0\rangle+i|1\rangle)}{\sqrt{2}}|00\rangle$. As it passes through M1, M2 and bomb effect part we get $\frac{(-|0\rangle+i|1\rangle)}{\sqrt{2}}|00\rangle$. On passing the photon through BS2 i.e., applying the U3 gate on the first qubit $q_{0}$ we get - $|000\rangle$ state. From the final result, it is observed that, the state of the photon at the end is found to be $|00\rangle$ state, which means it will be detected at the detector D1. The detailed calculation can be found in Appendix Section A.1.1. From the histogram (Fig. 4), it can be seen that the probability of $|00\rangle$ is 1 , which confirms the theoretical prediction. 


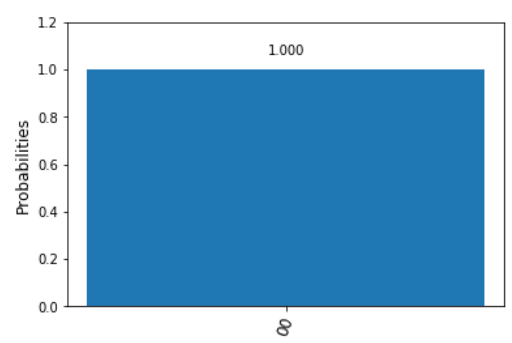

Figure 4. Histogram for the case when the input is from vertical direction and only D1 clicks, i.e., the probability of $|00\rangle$ is 1 .

\section{When direction of input photon is horizontal}

The input of photon is from horizontal direction (by convention), which is taken to be $|10\rangle$ as shown in Fig. 5. As the photon is in the horizontal direction and the bomb is dead, the state of the whole system is given as $|100\rangle$. On passing the photon through BS1 i.e. applying the U3 gate on the first qubit $q_{0}$ we get, $\frac{(|1\rangle+i|0\rangle)}{\sqrt{2}}|00\rangle$. As it passes through M1, M2 and bomb effect part we get, $\frac{(-|1\rangle+i|0\rangle)}{\sqrt{2}}|00\rangle$. On passing the photon through BS2 i.e. applying the U3 gate on the first qubit $q_{0}$ we get, $-|100\rangle$. The final state of the photon is calculated to be $|10\rangle$, which implies the photon will be detected at the detector D2. The histogram showing the probability of $|10\rangle$ is illustrated in the Fig. 7, that confirms the expected result. The detailed calculation of the quantum circuit shown in Fig. 6 can be found in Appendix Section A.1.2. The optical setup depicting the above scenario is presented in the Fig. 5.

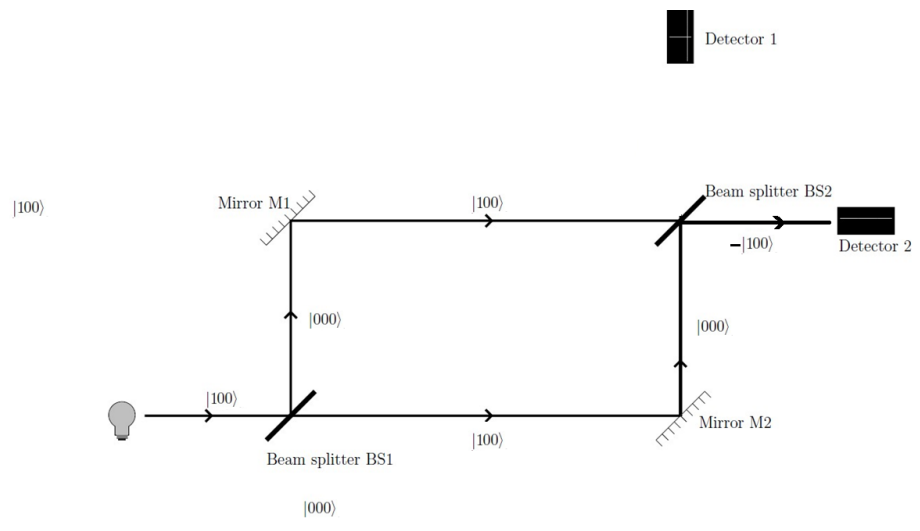

Figure 5. Setup for the cases when the bomb is not alive and the input is from horizontal direction. In this case D2 will click and D1 will not click. Constructive interference will occur at D2 while destructive interference at D1.

\section{CASE-2: When the bomb is there}

\section{When the input photon is from vertical direction}

The input of photon is from vertical direction (by convention), which is taken to be $|00\rangle$ state as shown in Fig. 8. As the bomb is there and the photon is from vertical direction, the state of the whole system is $|001\rangle$. On passing the photon through BS1 i.e. applying the U3 gate on the first qubit $q_{0}$ we get, $\frac{(|0\rangle+i|1\rangle)}{\sqrt{2}}|01\rangle$. On applying the mirrors M1, M2 and bomb effect part we get, $-\frac{(1-i \sqrt{2})|001\rangle+(i-\sqrt{2})|101\rangle+|011\rangle-i|111\rangle}{2 \sqrt{2}}$. The resultant state on applying BS2 is $-\frac{|00\rangle+|01\rangle}{2}-\frac{|10\rangle}{\sqrt{2}}|1\rangle$. The detailed calculation is available in Appendix Section A.2.1. As it can be seen, the final state of the photon is the superposition of $|00\rangle,|01\rangle$ and $|10\rangle$ with probabilities (Fig. 10) $0.25,0.25$ and 0.5 respectively, which represent the detection of photon at the detectors D1, D2 with 0.25 and 0.25 probabilities. It is 


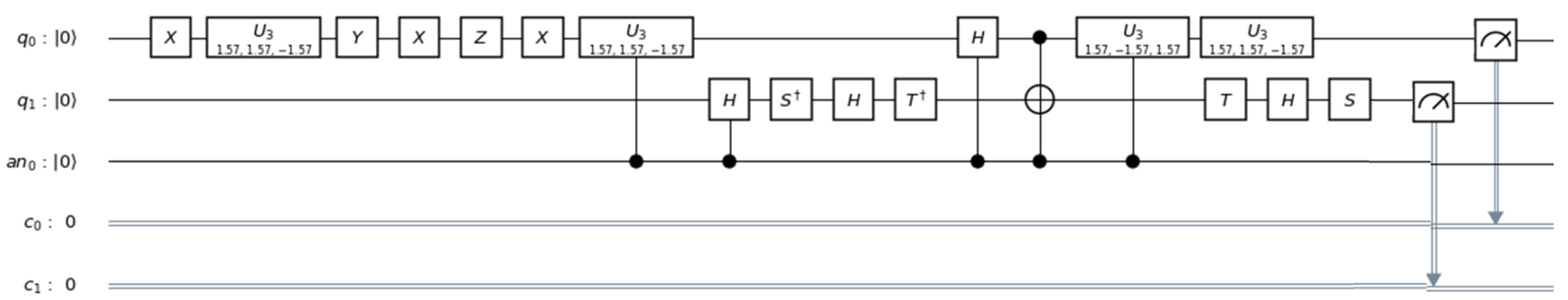

Figure 6. Quantum circuit for the cases when the bomb is not alive and the input is from horizontal direction. $X$ gate is applied on the qubit $q_{0}$ to denote the horizontal direction of the photon and the qubit $a n_{0}$ in state $|0\rangle$ represents the absence of the bomb.

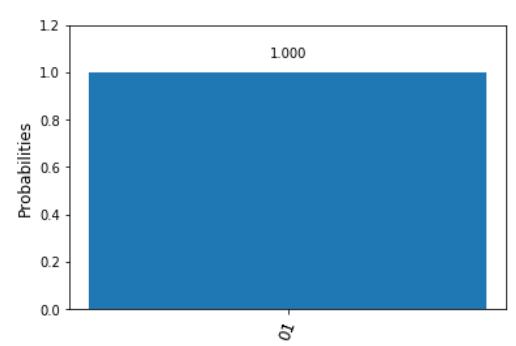

Figure 7. Histogram for the cases when the input is from horizontal direction, bomb is not alive then D2 clicks.

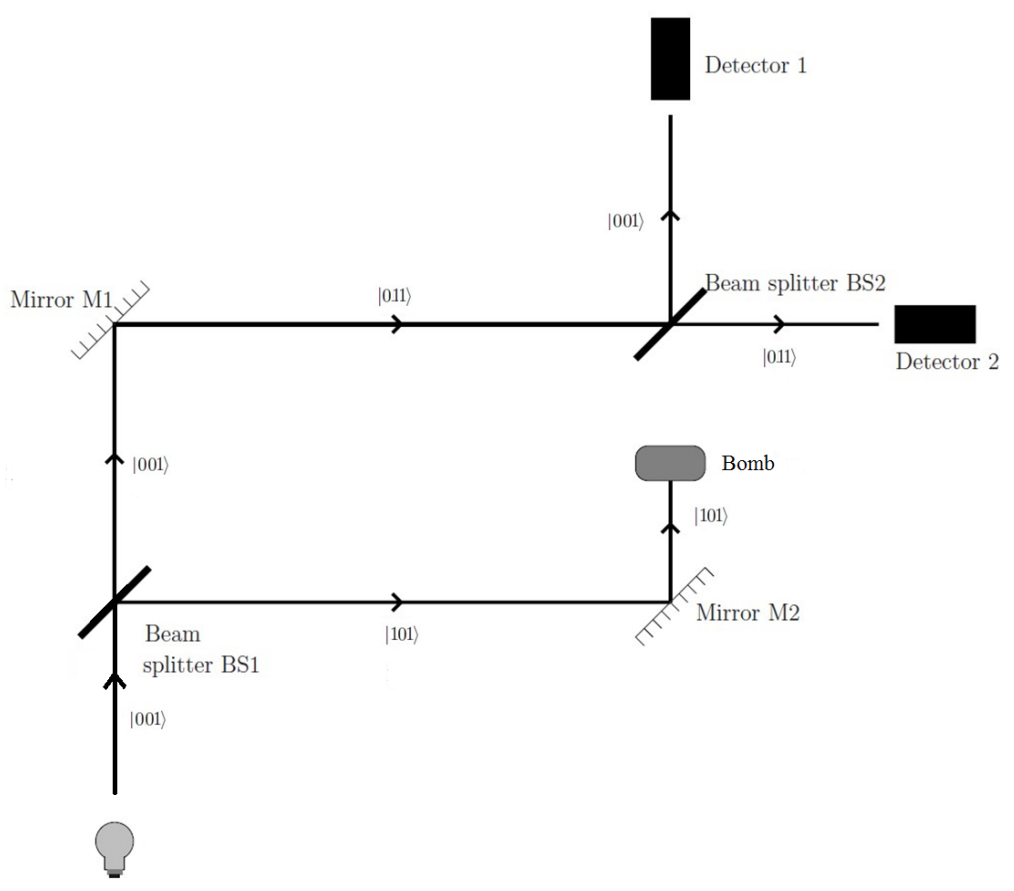

Figure 8. Quantum circuit illustrating the case when the bomb is alive and the input photon is from vertical direction. $X$ gate is applied and on the qubit $a n_{0}$ to signify the presence of the bomb. 


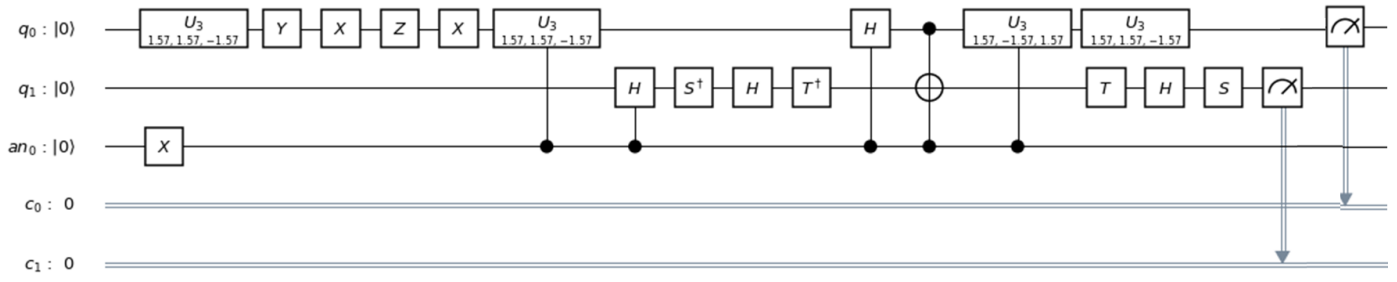

Figure 9. Setup for the cases when the bomb is alive and the input is from vertical direction. If the photon chooses lower path then explosion will occur (50\% of the cases) and when it chooses the upper path either of the detector (D1 or D2) clicks with 25-25\% probability.

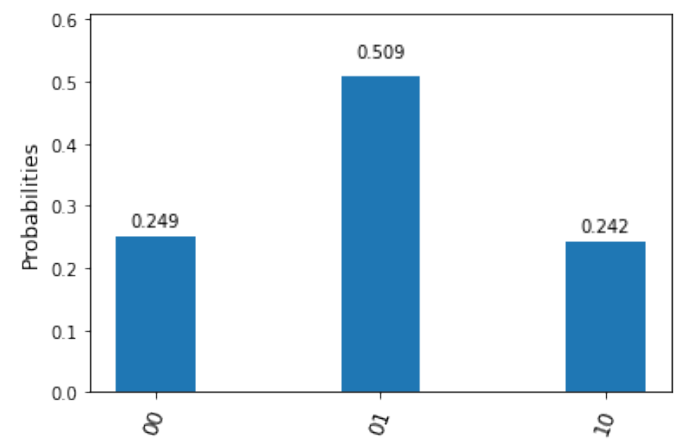

Figure 10. Histogram for the case when the bomb is alive and the input photon is from horizontal direction. The outcome's digit should be read from upper to lower i.e., the first, second and third bars of histogram represent the outcomes as $|00\rangle,|10\rangle,|01\rangle$ respectively.

observed that the photon with 0.5 probability, is not detected anywhere, which is denoted by the state $|10\rangle$ state.

\section{When input photon is from horizontal direction}

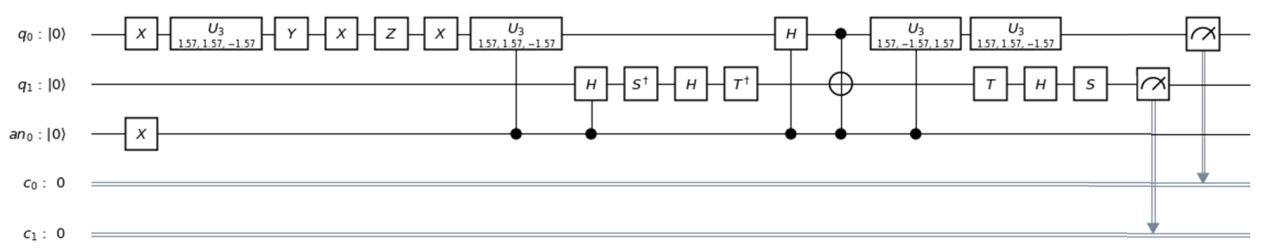

Figure 12. Setup for the case when the bomb is alive and input is from horizontal direction. If the photon chooses lower path then explosion will occur (50\% of the cases) and when it chooses the upper path either of the detector (D1 or D2) clicks with 25-25\% probability.

The input of photon (from horizontal direction) is taken to be $|10\rangle$ state as shown in Fig. 11 and due to the presence of bomb, the state of the whole system is $|101\rangle$. On passing the photon through BS1 i.e., applying the $\mathrm{U} 3$ gate on the first qubit $q_{0}$ we get $\frac{(|1\rangle+i|0\rangle)}{\sqrt{2}}|01\rangle$. On applying the mirrors M1, M2 and bomb effect part we get, $-\frac{(1+i \sqrt{2})|001\rangle+(i+\sqrt{2})|101\rangle+|011\rangle-i|111\rangle}{2 \sqrt{2}}$. The resultant state after applying BS2 is $-\frac{|00\rangle+|01\rangle}{2}+\frac{|10\rangle}{\sqrt{2}}|1\rangle$. The detailed calculation is provided in the Appendix Section A.2.2. The result can be verified from the histograms presented in Fig. 13, where the probabilities of $|00\rangle$, $|01\rangle$ and $|10\rangle$ are $0.25,0.25$ and 0.5 respectively. The outcomes $|00\rangle$ and $|01\rangle$ with each 0.25 probability represent the detection of the photon at the two detectors D1 and D2. The outcome $|10\rangle$ with 0.5 probability means the bomb will explode in $50 \%$ of the cases. 


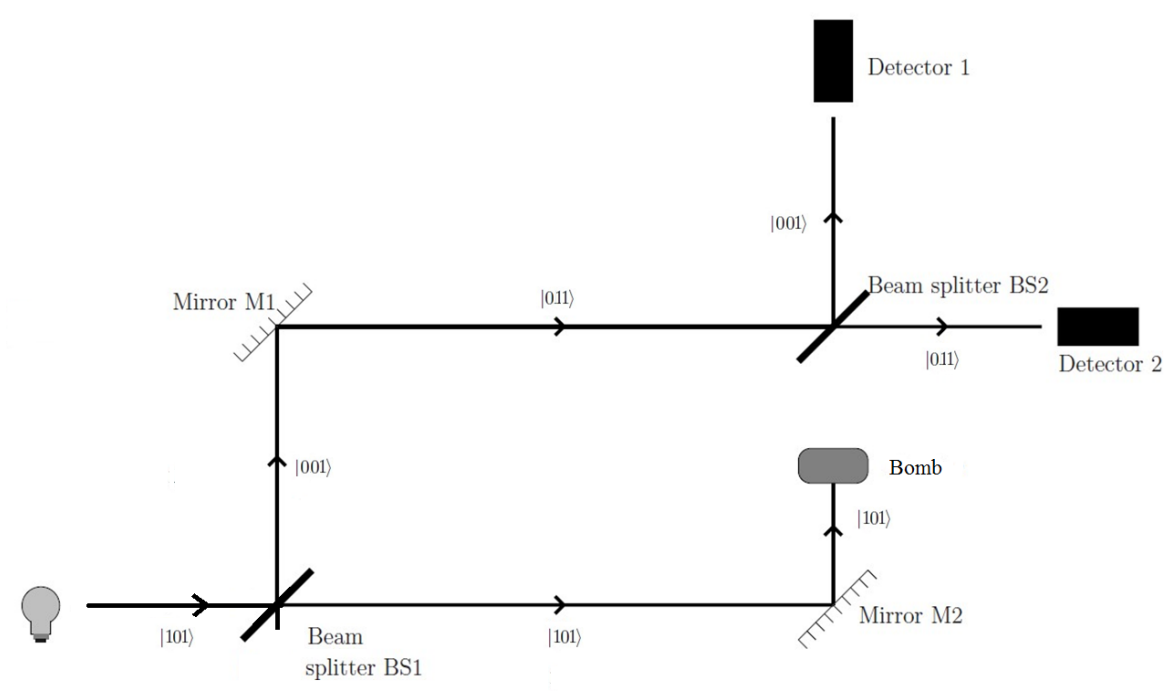

Figure 11. Quantum circuit illustrating the case when the bomb is alive and the input is from horizontal direction. X gates are applied on the qubits $q_{0}$ and $a n_{0}$ to represent the horizntal direction of the photon and the presence of bomb respectively.

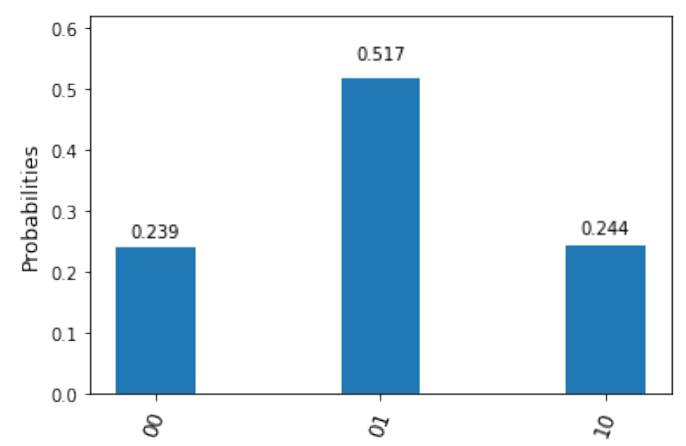

Figure 13. Histogram for the case when the bomb is alive and input is from vertical direction. As mentioned earlier, the outcomes from first to third are to be read as $|00\rangle,|10\rangle,|01\rangle$ respectively. 


\section{Conclusions}

To conclude, we have experimentally demonstrated here the realization of the EV IFM experiment as proposed by Elitzur and Vaidman [15] using quantum computation [46]. We have proposed new quantum circuits by designing them on the 5-qubit quantum chip, "ibmqx4" using QISKit as provided by IBM QE. We have then run the quantum circuits for different cases and verified the experimental results. It is observed that we can tell certainly that the bomb is alive in $25 \%$ cases without exploding it. The term "IFM" needs to be clarified here, as we cannot say in all the cases whether the bomb is alive or dead with certainty because there is $50 \%$ probability that the bomb will explode (i.e., the observer is interacting with the system) when it is alive. Also out of the rest $50 \%$ cases, in $25 \%$ of the cases we do not know if the bomb is live or dead. In the near future, the present work can be extended to demonstrate the Kwait et al. experiment [1] using quantum computation which can achieve IFM with almost $100 \%$ efficiency.

Author Contributions: Data Collection and Analysis, A.R. and B.D.; Scheme Analysis, A.R., B.D. and B.K.B.; Circuit Design, A.R., B.D. and B.K.B.; Investigation, A.R., B.D. and B.K.B.; Methodology, A.R. and B.D.; Project Administration, B.K.B.; Resources, A.R. and B.D.; Computations, A.R. and B.D.; Validation, A.R, B.D. and B.K.B.; Visualization, A.R., B.D. and B.K.B; Supervision, B.K.B. and P.K.P.; Writing-Original draft, A.R. and B.D.; Writing-Review and Editing, B.K.B. and P.K.P.

Funding: B.K.B. acknowledges the financial support of IISER Kolkata.

Acknowledgments: We acknowledge the hospitality of Indian Institute of Science Education and Research Kolkata during the project work. We thank Yugojyoti Mohanta (IISER Berhampur) for helping us avail QISKit. We acknowledge the support of IBM Quantum Experience for using the quantum processors. The views expressed are those of the authors and do not reflect the official position of IBM or the IBM quantum experience team.

Conflicts of Interest: The authors declare no conflict of interest.

\section{Appendix A. Equations involved in mirrors M1 and M2}

Appendix A.1. When the bomb is absent

Appendix A.1.1. Path is vertical

On applying operations of mirrors and bomb effect part one by one we get

a. Y gate on $q_{0} \rightarrow \frac{(|0\rangle+i|1\rangle)|00\rangle}{\sqrt{2}}$

b. X gate on $q_{0} \rightarrow \frac{(|1\rangle+i|0\rangle)|00\rangle}{\sqrt{2}}$

c. Z gate on $q_{0} \rightarrow \frac{(-|1\rangle+i|0\rangle)|00\rangle}{\sqrt{2}}$

d. X gate on $q_{0} \rightarrow \frac{(-|0\rangle+i|1\rangle)|00\rangle}{\sqrt{2}}$

e. Controlled U3 gate on $q_{0}$ only if $a n_{0}$ is $|1\rangle \rightarrow \frac{(-|0\rangle+i|1\rangle)|00\rangle}{\sqrt{2}}$

f. Controlled H gate on $q_{0}$ only if $a n_{0}$ is $|1\rangle \rightarrow \frac{(-|0\rangle+i|1\rangle)|00\rangle}{\sqrt{2}}$

g. Controlled H gate on $q_{1}$ only if $a n_{0}$ is $|1\rangle \rightarrow \frac{(-|0\rangle+i|1\rangle)|00\rangle}{\sqrt{2}}$

h. $S^{\dagger}$ gate on $q_{1} \rightarrow \frac{(-|0\rangle+i|1\rangle)|00\rangle}{\sqrt{2}}$

i. H gate on $q_{1} \rightarrow \frac{(-|0\rangle+i|1\rangle)(|0\rangle+|1\rangle)|0\rangle}{\sqrt{2}}$

j. T+ gate on $q_{1} \rightarrow \frac{(i|10\rangle+(i+1)(|11\rangle-|00\rangle-(1-i)|01\rangle)|0\rangle}{2 \sqrt{2}}$ 
k. Controlled not Controlled gate on $q_{1}$ only if $a n_{0} \& q_{0}$ are $|1\rangle \rightarrow\left(i|10\rangle+\frac{(i \pm 1)}{\sqrt{2}}|11\rangle-|00\rangle-\right.$ $\left.\frac{(1-i)|01\rangle}{\sqrt{2}}\right) \frac{|0\rangle}{2}$

1. Controlled $U 3^{\dagger}$ gate on $q_{0}$ only if $a n_{0}$ is $|1\rangle \rightarrow\left(i|10\rangle+\frac{(i+1)|11\rangle}{\sqrt{2}}-|00\rangle-\frac{(1-i)|01\rangle}{\sqrt{2}}\right) \frac{|0\rangle}{2}$

m. T gate on $q_{1} \rightarrow \frac{(-|0\rangle+i|1\rangle)(|0\rangle+|1\rangle)|0\rangle}{2}$

n. H gate on $q_{1} \rightarrow \frac{(-|0\rangle+i|1\rangle)|00\rangle}{\sqrt{2}}$

o. S gate on $q_{1} \rightarrow \frac{(|0\rangle+i|1\rangle)|00\rangle}{\sqrt{2}}$

Appendix A.1.2. when the direction is horizontal

On applying the operations of mirrors and bomb effect part one by one we get
a. Y gate on $q_{0} \rightarrow \frac{(-|1\rangle-i|0\rangle)|00\rangle}{\sqrt{2}}$
b. X gate on $q_{0} \rightarrow \frac{(|0\rangle-i|1\rangle)|00\rangle}{\sqrt{2}}$
c. Z gate on $q_{0} \rightarrow \frac{(-|0\rangle+i|1\rangle)|00\rangle}{\sqrt{2}}$
d. X gate on $q_{0} \rightarrow \frac{(-|1\rangle+i|0\rangle)|00\rangle}{\sqrt{2}}$

e. Controlled U3 gate on $q_{0}$ only if $a n_{0}$ is $|1\rangle \rightarrow \frac{(-|1\rangle+i|0\rangle)|00\rangle}{\sqrt{2}}$

f. Controlled H gate on $q_{0}$ only if $a n_{0}$ is $|1\rangle \rightarrow \frac{(-|1\rangle+i|0\rangle)|00\rangle}{\sqrt{2}}$

g. Controlled $\mathrm{H}$ gate on $q_{1}$ only if $a n_{0}$ is $|1\rangle \rightarrow \frac{(-|1\rangle+i|0\rangle)|00\rangle}{\sqrt{2}}$

h. $S^{\dagger}$ gate on $q_{1} \rightarrow \frac{(-|1\rangle+i|0\rangle)|00\rangle}{\sqrt{2}}$

i. H gate on $q_{1} \rightarrow \frac{(-|1\rangle+i|0\rangle)(|0\rangle+|1\rangle)|0\rangle}{\sqrt{2}}$

j. $T^{\dagger}$ gate on $q_{1} \rightarrow \frac{(i|00\rangle+(i+1)(|01\rangle-|10\rangle-(1-i)|11\rangle)|0\rangle}{2 \sqrt{2}}$

k. Controlled not Controlled gate on $q_{1}$ only if $a n_{0} \& q_{0}$ are $|1\rangle \rightarrow\left(i|00\rangle+\frac{(i+1)|01\rangle}{\sqrt{2}}-|10\rangle-\right.$ $\left.\frac{(1-i)|11\rangle}{\sqrt{2}}\right) \frac{|0\rangle}{2}$

m. T gate on $q_{1} \rightarrow \frac{(-|1\rangle+i|0\rangle)(|0\rangle+|1\rangle)|0\rangle}{2}$

n. H gate on $q_{1} \rightarrow \frac{(-|1\rangle+i|0\rangle)|00\rangle}{\sqrt{2}}$

o. S gate on $q_{1} \rightarrow \frac{(-|1\rangle+i|0\rangle)|00\rangle}{\sqrt{2}}$ 
Appendix A.2. when the bomb is present

Appendix A.2.1. Path is vertical

On applying operations for mirror and bomb effect part one by one we get
a. Y gate on $q_{0} \rightarrow \frac{(|0\rangle+i|1\rangle)|01\rangle}{\sqrt{2}}$
b. X gate on $q_{0} \rightarrow \frac{(|1\rangle+i|0\rangle)|01\rangle}{\sqrt{2}}$
c. Z gate on $q_{0} \rightarrow \frac{(-|1\rangle+i|0\rangle)|01\rangle}{\sqrt{2}}$
d. X gate on $q_{0} \rightarrow \frac{(-|0\rangle+i|1\rangle)|01\rangle}{\sqrt{2}}$

e. Controlled U3 gate on $q_{0}$ only if $a n_{0}$ is $|1\rangle \rightarrow-|001\rangle$

f. Controlled H gate on $q_{0}$ only if $a n_{0}$ is $|1\rangle \rightarrow \frac{(|0\rangle+|1\rangle)|01\rangle}{\sqrt{2}}$

g. Controlled H gate on $q_{1}$ only if $a n_{0}$ is $|1\rangle \rightarrow-\frac{(|0\rangle+|1\rangle)(|0\rangle+|1\rangle)|1\rangle}{2}$

h. S+ gate on $q_{1} \rightarrow-\frac{(|001\rangle-i|011\rangle+|101\rangle-i|111\rangle)}{2}$

i. H gate on $q_{1} \rightarrow-\frac{(1-i)|001\rangle+(1+i)|011\rangle+(1-i)|101\rangle+(1+i)|111\rangle}{2 \sqrt{2}}$

j. T+ gate on $q_{1} \rightarrow-\frac{(1-i)|001\rangle+\sqrt{2}|011\rangle+(1-i)|101\rangle+\sqrt{2}|111\rangle}{2 \sqrt{2}}$

k. Controlled not Controlled gate on $q_{1}$ only if $a n_{0} \quad \& \quad q_{0}$ are $|1\rangle \quad \rightarrow$ $-\frac{(1-i)|001\rangle+\sqrt{2}|011\rangle+(1-i)|111\rangle+\sqrt{2}|101\rangle}{2 \sqrt{2}}$

1. T gate on $\left.q_{1} \rightarrow-\frac{(1-i)|001\rangle+(1+i)|011\rangle+\sqrt{2}|101\rangle+\sqrt{2}|111\rangle}{2 \sqrt{2}}\right)$

m. H gate on $q_{1} \rightarrow-\frac{|001\rangle}{2}+\frac{i|011\rangle}{2}-\frac{|101\rangle}{\sqrt{2}}$

n. S gate on $q_{1} \rightarrow \frac{-|001\rangle}{2}+\frac{|011\rangle}{2}-\frac{|101\rangle}{\sqrt{2}}$

Appendix A.2.2. Path is horizontal

On applying the operations of mirrors and bomb effect part one by one we get

a. Y gate on $q_{0} \rightarrow \frac{(-|1\rangle-i|0\rangle)|01\rangle}{\sqrt{2}}$

b. X gate on $q_{0} \rightarrow \frac{(-|0\rangle-i|1\rangle)|01\rangle}{\sqrt{2}}$

c. Z gate on $q_{0} \rightarrow \frac{(-|0\rangle+i|1\rangle)|01\rangle}{\sqrt{2}}$

d. X gate on $q_{0} \rightarrow \frac{(-|1\rangle+i|0\rangle)|01\rangle}{\sqrt{2}}$

e. Controlled U3 gate on $q_{0}$ only if $a n_{0}$ is $|1\rangle \rightarrow-|101\rangle$ 
f. Controlled $\mathrm{H}$ gate on $q_{0}$ only if $a n_{0}$ is $|1\rangle \rightarrow-\frac{(|0\rangle-|1\rangle)|01\rangle}{\sqrt{2}}$

g. Controlled $\mathrm{H}$ gate on $q_{1}$ only if $a n_{0}$ is $|1\rangle \rightarrow-\frac{(|0\rangle-|1\rangle)(|0\rangle+|1\rangle)|1\rangle}{2}$

h. S+ gate on $q_{1} \rightarrow-\frac{|001\rangle-i|011\rangle-|101\rangle+i|111\rangle}{2}$

i. H gate on $q_{1} \rightarrow-\frac{(1-i)|001\rangle+(1+i)|011\rangle-(1-i)|101\rangle-(1+i)|111\rangle}{2 \sqrt{2}}$

j. T+ gate on $q_{1} \rightarrow-\frac{(1-i)|001\rangle+\sqrt{2}|011\rangle-(1-i)|101\rangle-\sqrt{2}|111\rangle}{2 \sqrt{2}}$

k. Controlled not Controlled gate on $q_{1}$ only if $a n_{0} \quad \& \quad q_{0}$ are $|1\rangle \quad \rightarrow$ $-\frac{(1-i)|001\rangle+|011\rangle-(1-i)|111\rangle-\sqrt{2}|101\rangle}{2 \sqrt{2}}$

1. T gate on $q_{1} \rightarrow-\frac{(1-i)|001\rangle+(1+i)|011\rangle-\sqrt{2}|101\rangle-\sqrt{2}|111\rangle}{2 \sqrt{2}}$

m. H gate on $q_{1} \rightarrow \frac{-|001\rangle}{2}+\frac{i|011\rangle}{2}+\frac{|101\rangle}{\sqrt{2}}$

n. S gate on $q_{1} \rightarrow \frac{-|001\rangle}{2}+\frac{|011\rangle}{2}+\frac{|101\rangle}{\sqrt{2}}$

\section{References}

1. Kwiat, P.; Interaction-Free Measurement Phys. Rev. Lett. 1995, 74, 4763.

2. Voorthuysen, E. H.; Realization of an interaction-free measurement of the presence of an object in a light beam. Am. J. Phys., 1996, 64, 1504.

3. Hafner, M.; Summhammer, J.; Experiment on interaction-free measurement in neutron interferometry. Phys. Lett. A. 1997, 235, 563

4. Tsegaye,T.; Goobar,E.; , Karlsson A.; Bjork ,G.; Loh, M. Y.; Lim, K. H.; Efficient interaction-free measurements in a high-finesse interferometer. Phys. Lett. A. 1998, 57, 3987.

5. White, A. G.; Mitchell, J. R., Nairz, O.; Kwiat, P.; “Interaction-free” imaging. Phys. Lett. A. 1998, 58, 605.

6. Kwiat, P.; Phys. Scripta 1998, 76, 115.

7. White, A. G.; Mitchell, J. R., Nairz, O.; Weihs, G.; Weinfurter,H.; and Zeilinger, A.; Kwiat, P.High-Efficiency Quantum Interrogation Measurements via the Quantum Zeno Effect. Phys. Rev. Lett. 1999, 83, 4725.

8. $\quad$ Mirell, S.; Mirell, D.; arXiv:quant-ph/0509028

9. Rudolph,T.; Better Schemes for Quantum Interrogation in Lossy Experiments. Phys. Rev. Lett. 2000, 85, 2925.

10. Elitzur, A. C.; Dolev, S.;Nonlocal effects of partial measurements and quantum erasure.Phys. Rev. A 2001, 63, 062109.

11. Potting, S.; Lee, E. S.; Schmitt, W.; Rumyantsev, I.; Mohring, B.; Meystre, P.; Quantum coherence and interaction-free measurements. Phys. Rev. A 2000, 62 060101(R).

12. Guo, G. C.; Guo Shi, B. S.; Quantum cryptography based on interaction-free measurement. Phys. Lett. A. 1999, 256109.

13. Czachor, M.; Quantum cryptography with polarizing interferometers. Phys. Lett. A. 1999, 257, 107.

14. Mitchison, G.;Jozsa, R.; Counterfactual computation.Proc. Royal Soc. A: Math. Phys. Eng. Sci. 2001, 457, 1175.

15. Elitzur, A. C.; Quantum mechanical interaction-free measurements. Found. Phys. 1993, 23, 987.

16. Vaidman, L.; The Meaning of the Interaction-Free Measurements. Found. Phys. 2003, 33, 491.

17. Pavicic, M.; Resonance energy-exchange-free detection and "welcher Weg" experiment. Phys. Rev. A1996, 223, 241.

18. IBM Quantum Experience, URL: https:/ / quantumexperience.ng.bluemix.net/qx/community.

19. QISKit, URL: https://github.com/Qiskit/qiskit-terra.

20. Jupyter Notebook, URL: https://jupyter.org/.

21. Dutta, S.; Suau, A.; Dutta, S.; Roy, S.; Behera, B. K.; and Panigrahi, P. K. Demonstration of a Quantum Circuit Design Methodology for Multiple Regression. arXiv:1811.01726. 
22. Woerner, S.; Egger, D. J. Quantum Risk Analysis. arXiv:1806.06893.

23. Viyuela, O.; Rivas, A.; Gasparinetti, S.; Wallraff, A.; Filipp, S.; Martin-Delgado, M. A. npj Quantum Inf. 2018, 4,10 .

24. Aggarwal, D.; Raj, S.; Behera, B. K.; and Panigrahi, P. K. Application of quantum scrambling in Rydberg atom on IBM quantum computer. arXiv:1806.00781.

25. Zhukov, A. A.; Remizov, S. V.; Pogosov, W. V.; Lozovik, Y. E. Algorithmic simulation of far-from-equilibrium dynamics using quantum computer. Quantum Inf. Process. 2018, 17, 223.

26. Hegade, N. N.; Behera, B. K.; and Panigrahi, P. K. Experimental Demonstration of Quantum Tunneling in IBM Quantum Computer. arXiv:1712.07326.

27. Halder, K.; Hegade, N. N.; Behera, B. K.; Panigrahi, P. K. Digital Quantum Simulation of Laser-Pulse Induced Tunneling Mechanism in Chemical Isomerization Reaction. arXiv:1808.00021.

28. Malik, G. R.; Singh, R. P.; Behera, B. K.; Panigrahi, P. K. First Experimental Demonstration of Multi-particle Quan-tum Tunneling in IBM Quantum Computer. DOI: 10.13140/RG.2.2.27260.18569.

29. Vuillot, C. Is error detection helpful on IBM 5Q chips?. Quantum Inf. Comput. 2018, 18, 0949

30. Ghosh, D.; Agarwal, P.; Pandey, P.; Behera, B. K.; and Panigrahi, P. K. Automated error correction in IBM quantum computer and explicit generalization. Quantum Inf. Process. 2018, 17, 153.

31. Singh, R. K.; Panda, B.; Behera, B. K.; and Panigrahi, P. K. Demonstration of a general fault-tolerant quantum error detection code for (2n+1)-qubit entangled state on IBM 16-qubit quantum computer. arXiv:1807.02883.

32. Satyajit, S.; Srinivasan, K.; Behera, B. K.; and Panigrahi, P. K. Nondestructive discrimination of a new family of highly entangled states in IBM quantum computer. Quantum Inf. Process. 2018, 17, 212.

33. Behera, B. K.; Banerjee, A.; Panigrahi, P. K. Experimental realization of quantum cheque using a five-qubit quantum computer. Quantum Inf. Process. 2017, 16, 312.

34. Biswas, S.; Razdan, S.; Behera, B. K.; and Panigrahi, P. K. Realization of Counterfactual Quantum Cryptography Us-ing IBM's Quantum Computer. DOI: 10.13140/RG.2.2.30090.52160.

35. Alsina, D.; Latorre, J. I. Experimental test of Mermin inequalities on a five-qubit quantum computer. Phys. Rev. A 2016, 94, 012314.

36. Kalra, A. R.; Gupta, N.; Behera, B. K.; Prakash, S.; Panigrahi, P. K. Demonstration of the No-Hiding Theorem on the 5 Qubit IBM Quantum Computer in a Category Theoretic Framework. arXiv:1707.09462.

37. Swain, M.; Rai, A.; Behera, B. K.; and Panigrahi, P. K. Experimental demonstration of the violations of Mermin's and Svetlichny's inequalities for W- and GHZ-class of states. arXiv:1810.00874.

38. Singh, R. P.; Behera, B. K.; and Panigrahi, P. K. Quantum algorithm for sum of infinite series; determining the value of $\pi$. DOI: $10.13140 /$ RG.2.2.21343.10408.

39. Gangopadhyay, S.; Manabputra; Behera, B. K.; and Panigrahi, P. K. Generalization and demonstration of an entanglement-based Deutsch-Jozsa-like algorithm using a 5-qubit quantum computer. Quantum Inf. Process. 2018, 17, 160

40. Srinivasan, K.; Satyajit, S.; Behera, B. K.; and Panigrahi, P. K. Efficient quantum algorithm for solving travelling salesman problem: An IBM quantum experience. arXiv:1805.10928.

41. Pal, A.; Chandra, S.; Mongia, V.; B. K. Behera and Panigrahi, P. K. Solving Sudoku Game Using Quantum Computation. DOI: 10.13140/RG.2.2.19777.86885.

42. Roy, B. B.; Behera, B. K.; and Panigrahi, P. K. Modelling A Simple Shooting Game Using Quantum Computation. DOI: 10.13140/RG.2.2.30976.07680.

43. Paul, S.; Behera, B. K.; Panigrahi, P. K. Playing Quantum Monty Hall Game in a Quantum Computer. arXiv:1901.01136.

44. Behera, B. K.; Reza, T.; Gupta, A.; Panigrahi, P. K. Designing Quantum Router in IBM Quantum Computer. arXiv:1803.06530. 2018

45. Behera, B. K.; Seth, S.; Das, A.; Panigrahi, P. K.;arXiv:1712.00854.

46. Nielsen, M. A.; Chuang, I.; Grover, L. K.; Quantum Computation and Quantum Information. Am. J. Phys. 2002, 70, 558. 